\title{
Gastrointestinal stromal tumor penetrating into the small intestine in a patient with neurofibromatosis type 1
}

\author{
Hanae Kobayashi • Yoshito Ikematsu • \\ Hiroki Mori · Takachika Ozawa • Taketoshi Okawada • \\ Yoshiro Nishiwaki
}

Received: 12 March 2012/Accepted: 29 January 2013/Published online: 23 February 2013

(C) The Japan Society of Clinical Oncology 2013

\begin{abstract}
A 53-year-old woman with neurofibromatosis type 1 (NF-1) suddenly experienced left lower abdominal pain. Next day she was referred to our hospital for further examinations because her white blood cell count and C-reactive protein levels were elevated. Computed tomography revealed a $>10 \mathrm{~cm}$ mass in the mid-abdomen. The mass contained air-fluid level material encapsulated by a thickened wall. Because gas was observed in the tumor, a fistula was suspected between the abscess in the mass and the small intestine. The association of the tumor and NF-1 indicated that the mass was a gastrointestinal stromal tumor arising from a part of the small intestine communicating with the gut, which resulted in abscess formation. An emergency operation was performed on the day of admission, and the tumor was removed by resecting the 2 intestinal loops by adhesiolysis. Few disseminated daughter nodules were also identified on the surface of the distant intestine. The patient was discharged from the hospital on
\end{abstract}

H. Kobayashi · Y. Ikematsu · Y. Nishiwaki

Department of Surgery, Hamamatsu Medical Center,

Hamamatsu, Japan

H. Kobayashi $(\square)$

Department of Breast Surgery, Hamamatsu Medical Center, 328 Tomitsuka, Hamamatsu, Shizuoka 432-8580, Japan

e-mail: hanae.kobayashi@hmedc.or.jp

H. Mori · T. Ozawa

Department of Pathology, Hamamatsu Medical Center,

Hamamatsu, Japan

T. Okawada

Department of Radiology, Hamamatsu Medical Center,

Hamamatsu, Japan the 10th postoperative day with no complications. Because of the remaining lesions, molecular targeted chemotherapy with $400 \mathrm{mg} /$ day imatinib was initiated. There has been no sign of progression in past 18 months.

Keywords GIST · Neurofibromatosis type1 - KIT · PDGFR $\alpha$

\section{Introduction}

Neurofibromatosis type 1 (NF-1), or von Recklinghausen disease, is an autosomal dominant inherited disease caused by a mutation in the NF-1 gene on the 17 th chromosome. However, half of patients with the disease do not have a family history of NF-1, and sporadic mutation on the target site is believed to be of crucial importance in NF-1 pathogenesis [1]. NF-1 gene mutation results in many types of neural tumor, and skin pigmentation referred to as caféau-lait spot on the entire body surface. NF-1 patients are at an increased risk of developing malignant tumors, particularly non-epithelial tumors in the gastrointestinal system. The incidence of non-epithelial gastrointestinal stromal tumors (GIST) in NF-1 patients is 7-25\%, and, unlike common GISTs, they predominantly grow from the small intestine [2-4]. In cases with NF-1-associated GISTs, cautious examination and accurate diagnosis are needed when a patient presents with abdominal pain and subsequent gastrointestinal bleeding. Also, incidence of mutant c-kit and platelet-derived growth factor receptor $\alpha$ (PDGFR $\alpha$ ) in NF-1-related GISTs is known to be lower than in sporadic GISTs (11.5 vs. $85 \%$ ), which have poor response to molecular therapy. Postoperative treatment of non-c-kit/PDGFR $\alpha$ mutational GIST, including adjuvant therapy, is still controversial [14]. 


\section{Case report}

A 53-year-old woman with NF-1 suddenly experienced left lower abdominal pain without any prodromes on December 7, 2010. Next day she visited a local physician. Her white blood cell count and C-reactive protein levels were elevated, and she was referred to our hospital on the same day. Her body temperature was $38.0^{\circ} \mathrm{C}$, and physical examination revealed slight abdominal distension and signs of local peritoneal irritation in the left lower quadrant. Several soft skin tumors and café-au-lait spots due to NF-1 were observed over the entire body. She had an incision scar from a caesarean section in the lower mid-abdomen. Complete blood counts showed: white blood cell count, 13,800/ $\mathrm{mm}^{3}$; red blood cell count, $4.25 \times 10^{6} / \mathrm{mm}^{3}$; hemoglobin, $12.6 \mathrm{~g} /$ $\mathrm{dl}$; hematocrit, $38.1 \%$; and platelet count, $224,000 / \mathrm{mm}^{3}$. Serum chemistry showed C-reactive protein of $20.15 \mathrm{mg} / \mathrm{dl}$ (normal, <0.27). Elevation of aspartate aminotransferase (AST), 69 IU/l; alanine aminotransferase (ALT), 127 IU/l; alkaline phosphatase (ALP), 1077 IU/l; and gamma guanosine-5'-triphosphate $(\gamma \mathrm{GTP}), 295 \mathrm{IU} / 1$ indicated slight hepatic dysfunction. The tumor markers carcinoembryonic antigen (CEA) and carbohydrate antigen 19-9 (CA19-9) were within normal limits.

An abdominal computed tomography (CT) scan revealed a huge $(13-\mathrm{cm})$ mass in the middle of the abdominal cavity (Fig. 1). The mass had a thickened capsule, gas, and fluid. Typical air-fluid content suggested fistula formation between the intestine and the tumor. Local inflammation had increased the fat density around the mass, but ascites were not detected in the abdomen. Because the patient had NF-1, the first differential diagnosis for the acute abdominal pain was a GIST from the small intestine which had asymptomatic growth, became necrotic, underwent denaturation, and penetrated the intestine. Because of the signs of peritoneal irritation, which is reliable evidence of peritonitis, and high inflammatory responses in the blood and serum, we decided to perform an emergency operation on the day of admission. Tumor excision was performed with partial combined resection of 2 jejunal loops. During the operation, several small soft nodules on the peritoneal surface were identified as seeds (Fig. 2). One of the disseminated nodules was inside the resected specimen. The patient was discharged from the hospital on the 10th postoperative day without any complications.

Macroscopic examination revealed a 13-cm-wide tumor and the 2 loops of the small intestine were attached to each other. The tumor was attached to the 2 loops; one was just adhesion and the other had a pinhole-sized fistula (Fig. 3). The tumor was a huge cyst that contained denatured liquid (or pus). Some parts of the abscess wall had white mural nodules, which suggested residual viable tumor cells. A few small disseminated satellite lesions were observed on the surface of the intestine, close to the main tumor. Microscopically, the tumor was a single cyst showing coagulation necrosis and contained an abscess. Hyperplasia of the spindle-shaped cells was observed in the cyst wall, particularly in the muscular tunic near the fistula, which was assumed to be the site of tumor origin (Fig. 4). Mitosis was observed in 2 cells only in 50 high-power fields (HPF) (Fig. 5). The results of immunohistochemical staining were positive for CD117, DOG-1, CD34, and vimentin, and negative for smooth muscle actin (SMA), desmin, and S-100, which led to diagnosis of GIST from the small intestine. The satellite lesions on the jejunum were histologically identical with the main tumor. Molecular examination of the main tumor by polymerase chain reaction and direct sequencing revealed no mutations in exons 9, 11, 13, and 17 of the c-kit gene, and in exons 12 and 18 of the PDGFR $\alpha$ gene. Although they were histologically
Fig. 1 Computed tomography (CT) images taken on the day of admission. a Axial image of abdomen, showing a tumor containing air-fluid level material (white arrowheads show gas inside). b Coronal image of the abdomen
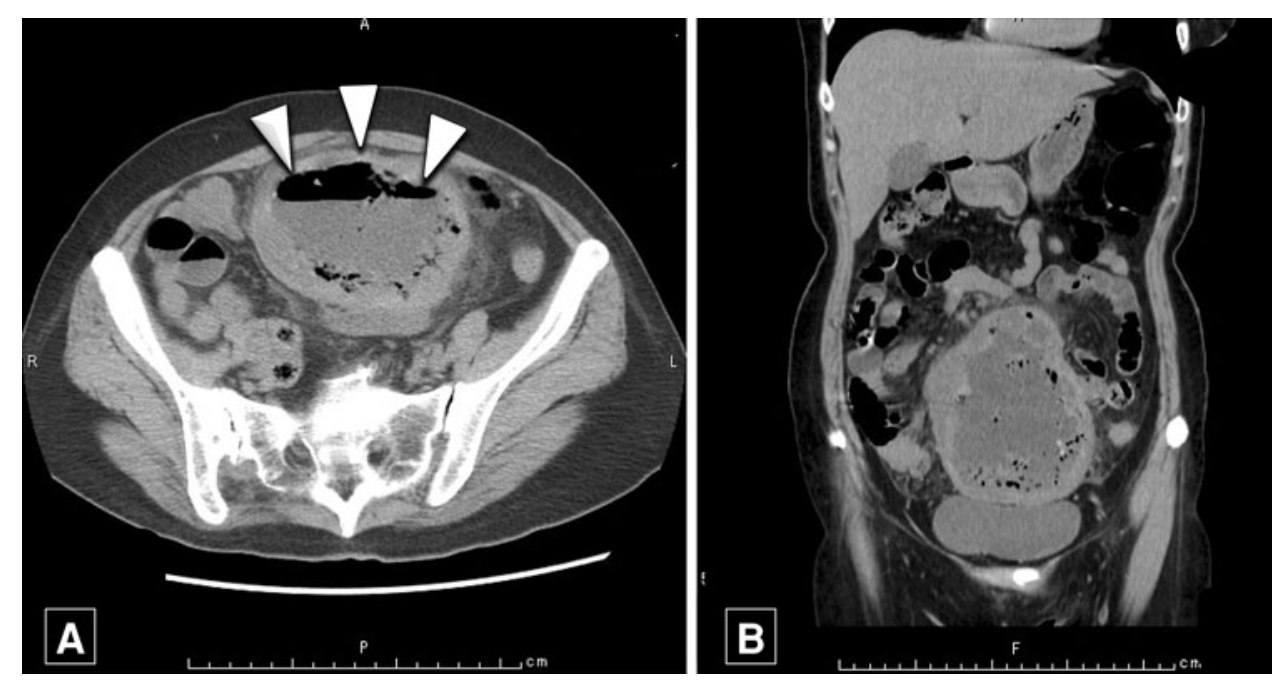
Fig. 2 Intraoperative findings. a A large white encapsulated tumor attached to 2 jejunal loops (black arrowheads show the point of attachment of the jejunum with the fistula). b A small disseminated satellite lesion (white arrowheads) close to the main tumor
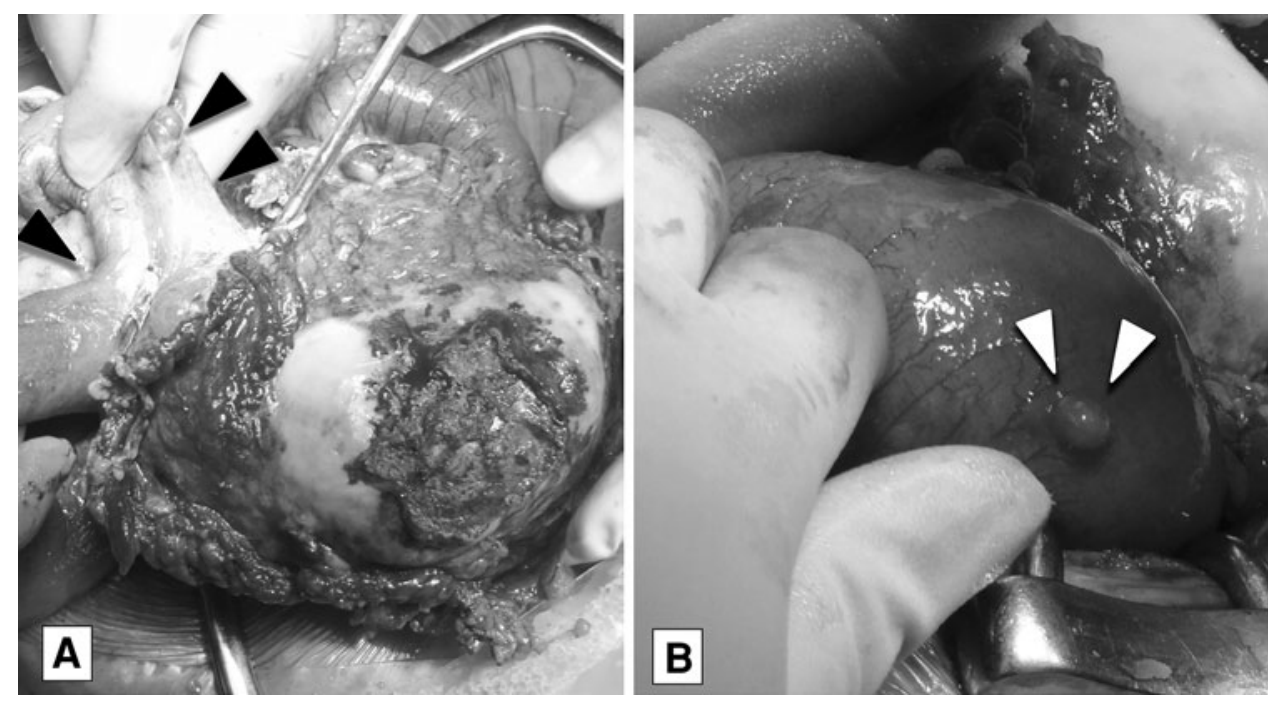

Fig. 3 Macroscopic findings. The tumor consisted of a single cyst showing coagulation necrosis and an abscess. The probe shows where the tumor and the jejunal loop communicate (white arrowhead). a The whole resected specimen (formalinfixed); b cut surface (formalinfixed)
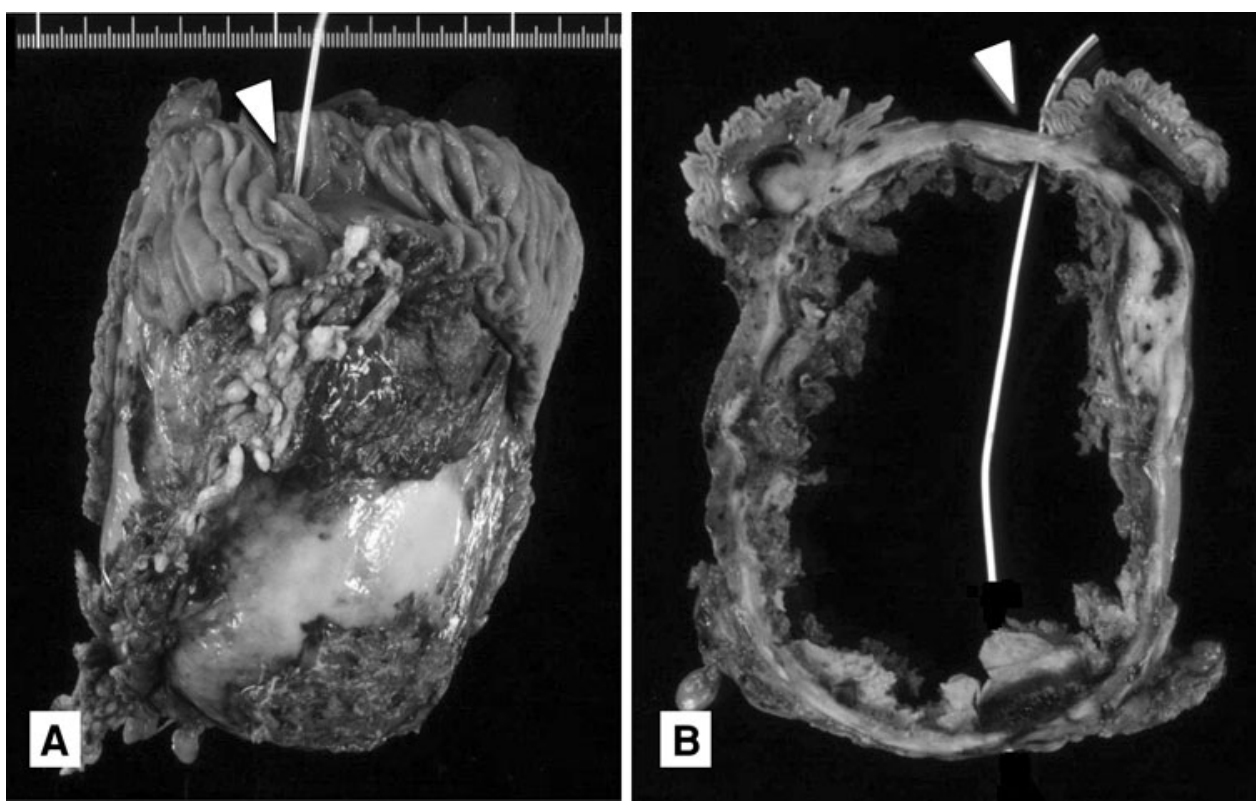

identical, we could not definitely determine whether the satellite nodules were dissemination or multifocal lesions.

There was concern about the efficacy of molecular targeted chemotherapy against intestinal GIST without mutation. However, because there were evident remnant lesions, we started $400 \mathrm{mg} /$ day imatinib for treatment. The patient has shown good recovery for 12 postoperative months and for 12 months after starting imatinib without any signs of recurrence.

\section{Discussion}

Neurofibromatosis type 1 is a common inherited disease, and incidence is 1 in 3,000 births [5]. One of the mechanisms most responsible for NF-1 is loss of function of the tumor suppressor gene on chromosome 17q 11.2, leading to the inactivation of neurofibromin, a negative regulator of the ras signal-transduction pathway [6-8]. Approximately $25 \%$ of NF-1 patients are known to be at increased risk of developing gastrointestinal non-epithelial tumors, for example neurofibroma, schwannoma, carcinoids, and GISTs [2]. The incidence of NF-1 cases associated with GISTs has recently been increasing. In Japan, 36 cases were been reported by ICHUSHI, a Japanese medical search engine, with keywords $N F-1$ and small intestine GIST. Among these cases, 9 patients were found as a result of melena, 9 had a different tumor (sigmoid colon cancer, pheochromocytoma, etc.), and 8 were multicentric. Among 25 reported cases of NF-1-associated GIST, $92 \%$ cases originated from the small intestine and in $60 \%$ cases it was multicentric [9]. 
Fig. 4 Pathological (loupe magnification) findings. a Hematoxylin-eosin-stained tissue of tumor origin. The tumor consisted of spindleshaped cells growing out from the muscular tunic of the intestinal wall (black arrowheads).

b Immunohistochemical staining of c-kit
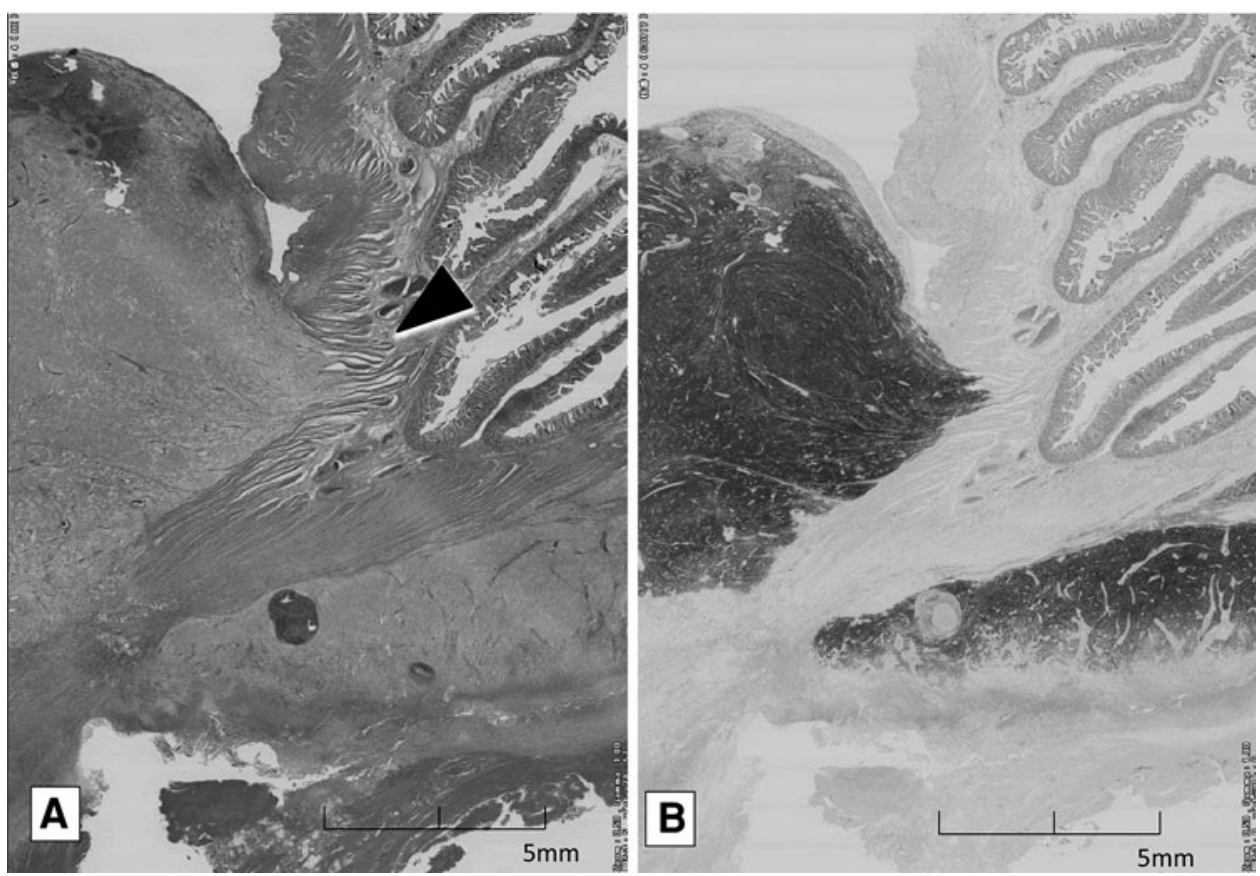

Fig. 5 Pathological $(\times 400)$ findings. a Hematoxylin-eosinstained tissue where mitosis is observed in 2 cells only in 50 high-power fields (HPF).

b Tumor tissue shows strong staining of c-kit
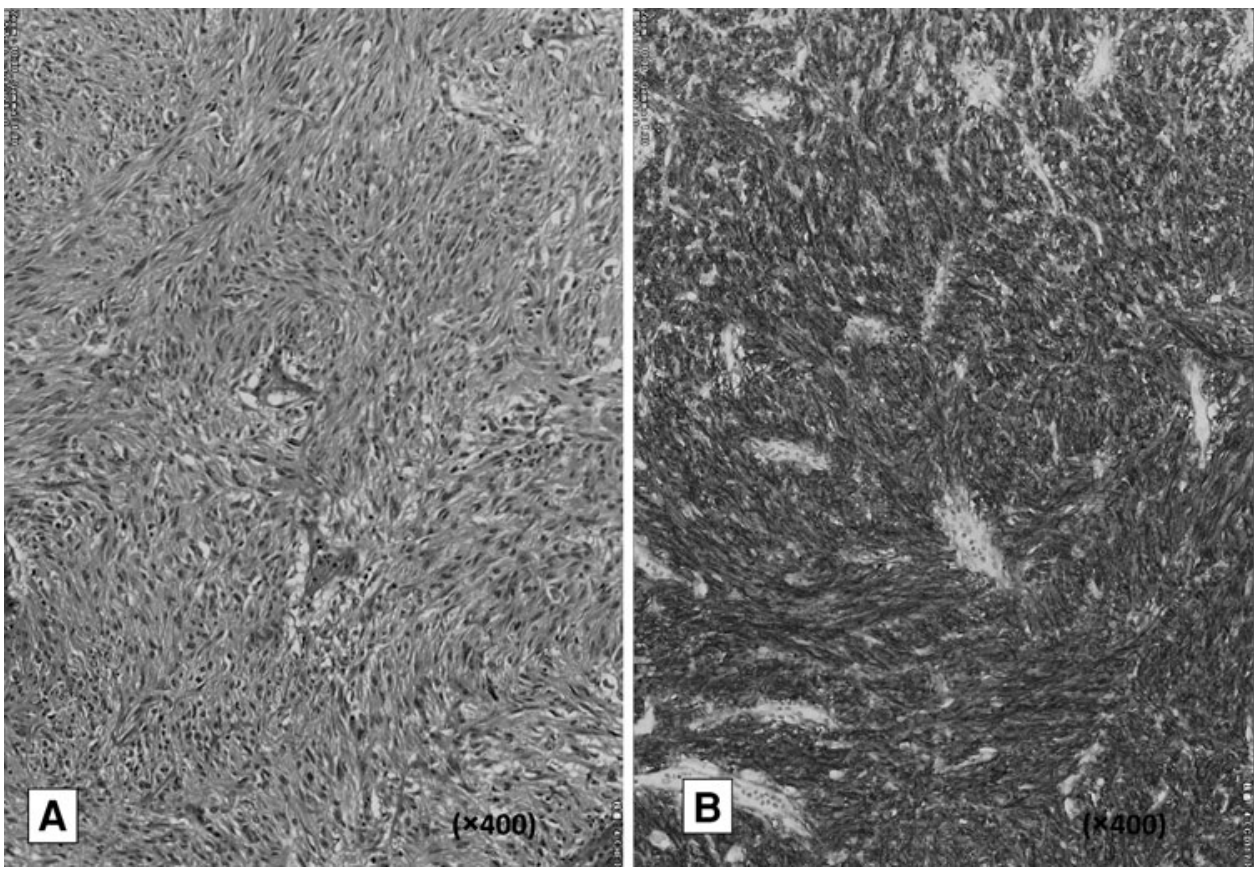

GIST is the most common mesenchymal neoplasm which develops from the gut. Cajal cells in the intestinal wall function as a pacemaker in peristalsis, and in the gastrointestinal tract only these cells have the c-kit gene and express both KIT and CD34 proteins. Because overexpression of these 2 proteins is a specific feature of most GISTs, Cajal cells are believed to be the origin of GISTs [10]. However, recent studies suggest that approximately $89 \%$ of GISTs in NF-1 patients do not have c-kit or PDGFR $\alpha$ mutations, and the underlying molecular mechanism is still being investigated [11, 12]. As previously described, neurofibromin caused by loss of NF-1 gene function is major pathology of NF-1, and leads to deregulation of the p21(ras) pathway and Fas protein. Constitutive activation of ras and increased mitogen-activated protein kinase (MAP kinase) signaling and reduced apoptosis contribute to tumor development [12, 13]. Because the generation pathway of NF-1-related GISTs is different from that of sporadic GISTs, and it is often nonc-kit/PDGFR $\alpha$ mutational, it was of concern whether 
molecular targeted chemotherapy would be worthwhile. Nevertheless, we decided to add imatinib by considering tumor malignancy and the presence of residual lesions.

In the adjuvant setting, GIST recurrence risks and its histological behavior are classified on the basis of its size, mitotic cell count, and origin [4, 15]. Our case had residual tumors, which did not meet adjuvant criteria, but its size and origin were indicative of malignant behavior despite the low mitotic aspect. In fact, it was controversial whether these residual tumors were disseminations or multifocal lesions, because it is known that GISTs develop multicentrically, particularly in patients with NF-1 [12]. However, because of the size discrepancy between main tumor and satellites, we decided to think of them as disseminations.

There is doubt whether imatinib affects non-c-kit/ PDGFR $\alpha$ mutational GIST. Mussi et al. reported cases of non-gene mutational GISTs with NF-1 treated by imatinib. In this report, no recurrence was seen in adjuvant cases, but aggressive progression was observed for all metastatic patients, indicating the lack of effectiveness of imatinib [14]. The mechanism is unclear, but we can hypothesize it is because activation of the MAP kinase pathway in GIST with NF-1 is not related to tyrosine kinase, which imatinib can inhibit. But our case showed high malignancy and imatinib was the only treatment we could add, so imatinib $400 \mathrm{mg} /$ day was prescribed.

Thorough follow-up of the patient is needed and similar cases should be accumulated to clarify the characteristics of this disease. Until sufficient evidence has been accumulated, we believe imatinib is worth trying even for nonc-kit/PDGFR $\alpha$ mutational GIST.

\section{Conclusions}

Because the number of NF-1 cases with intestinal GIST is limited, there is no established standard treatment for this condition. Different mechanisms are suspected for nonNF-1 GIST, and further investigation is needed about to avoid unnecessary molecular target therapy for such patients.

Conflict of interest The authors declare that they have no conflict of interest.

\section{References}

1. Ohno K (2010) Continuous clinical management of patients with neurofibromatosis type 1 (in Japanese). Nippon Rinsho 68: 131-135

2. Zoller ME, Rembeck B, Oden A et al (1997) Malignant and benign tumors in patients with neurofibromatosis type 1 in a defined Swedish population. Cancer 79:2125-2131

3. Fuller CE, Williams GT (1991) Gastrointestinal manifestations of type 1 neurofibromatosis (von Recklinghausen's disease). Histopathology 19:1-11

4. Miettinen M, Sobin LH, Sarlomo-Rikala M (2000) Immunohistochemical spectrum of GISTs at different sites and their differential diagnosis with a reference to CD117 (KIT). Mod Pathol 13:1134-1142

5. Friedman JM (1999) Epidemiology of neurofibromatosis type 1. Am J Med Genet 89:1-6

6. Wallace MR, Marchuk DA, Andersen LB et al (1990) Type 1 neurofibromatosis gene: identification of a large transcript disrupted in three NF1 patients. Science 249:181-186

7. Viskochil D, Buchberg AM, Xu G et al (1990) Deletions and a translocation interrupt a cloned gene at the neurofibromatosis type 1 locus. Cell 62:187-192

8. Basu TN, Gutmann DH, Fletcher JA et al (1992) Aberrant regulation of ras proteins in malignant tumour cells from type 1 neurofibromatosis patients. Nature 356:713-715

9. Iwasaki J, Yano T, Sanda T et al (2008) A case of multiple gastrointestinal stromal tumors (GISTs) of the jejunum associated with von Recklinghausen's disease (in Japanese). J Jpn Surg Assoc 69:1988-1992

10. Hirota S, Isozaki K, Moriyama Y et al (1998) Gain-of-function mutations of c-kit in human gastrointestinal stromal tumors. Science 279:577-580

11. Kinoshita K, Hirota S, Isozaki K et al (2004) Absence of c-kit gene mutations in gastrointestinal stromal tumours from neurofibromatosis type 1 patients. J Pathol 202:80-85

12. Andersson J, Sihto H, Meis-Kindblom JM et al (2005) NF1associated gastrointestinal stromal tumors have unique clinical, phenotypic, and genotypic characteristics. Am J Surg Pathol 29:1170-1176

13. Hiatt K, Ingram DA et al (2004) Loss of the nf 1 tumor suppressor gene decreases fas antigen expression in myeloid cells. Am J Pathol 164(4):1471-1479

14. Mussi C, Schildhaus HU, Gronchi A et al (2008) Therapeutic consequences from molecular biology for gastrointestinal stromal tumor patients affected by neurofibromatosis type 1 . Clin Cancer Res 14:4550-4555

15. Fletcher CD, Berman JJ, Corless C et al (2002) Diagnosis of gastrointestinal stromal tumors: a consensus approach. Hum Pathol 33:459-465 